\title{
Cortical Tonotopic Map Changes in Humans Are Larger in Hearing Loss Than in Additional Tinnitus
}

\author{
${ }^{\circledR}$ Elouise A. Koops, ${ }^{1,2,3}$ Remco J. Renken, ${ }^{2,3}$ Cris P. Lanting, ${ }^{1}$ and ${ }^{\circledR}$ Pim van Dijk ${ }^{1,2,3}$ \\ ${ }^{1}$ Department of Otorhinolaryngology/Head and Neck Surgery, University of Groningen, University Medical Center Groningen, Groningen, 9700 RB, \\ The Netherlands, ${ }^{2}$ Graduate School of Medical Sciences (Research School of Behavioural and Cognitive Neurosciences), University of Groningen, \\ Groningen, $9713 \mathrm{AV}$, The Netherlands, and ${ }^{3}$ University of Groningen, Cognitive Neuroscience Center, Biomedical Sciences of Cells and Systems, \\ Groningen, $9713 \mathrm{AW}$, The Netherlands
}

Neural plasticity due to hearing loss results in tonotopic map changes. Several studies have suggested a relation between hearing loss-induced tonotopic reorganization and tinnitus. This large fMRI study on humans was intended to clarify the relations between hearing loss, tinnitus, and tonotopic reorganization. To determine the differential effect of hearing loss and tinnitus, both male and female participants with bilateral high-frequency hearing loss, with and without tinnitus, and a control group were included. In a total of 90 participants, bilateral cortical responses to sound stimulation were measured with loudness-matched pure-tone stimuli $(0.25-8 \mathrm{kHz})$. In the bilateral auditory cortices, the high-frequency sound-evoked activation level was higher in both hearing-impaired participant groups, compared with the control group. This was most prominent in the hearing loss group without tinnitus. Similarly, the tonotopic maps for the hearing loss without tinnitus group were significantly different from the controls, whereas the maps of those with tinnitus were not. These results show that higher response amplitudes and map reorganization are a characteristic of hearing loss, not of tinnitus. Both tonotopic maps and response amplitudes of tinnitus participants appear intermediate to the controls and hearing loss without tinnitus group. This observation suggests a connection between tinnitus and an incomplete form of central compensation to hearing loss, rather than excessive adaptation. One implication of this may be that treatments for tinnitus shift their focus toward enhancing the cortical plasticity, instead of reversing it.

Key words: ageing; auditory cortex; hearing loss; plasticity; tinnitus; tonotopy

Significance Statement

Tinnitus, a common and potentially devastating condition, is the presence of a "phantom" sound that often accompanies hearing loss. Hearing loss is known to induce plastic changes in cortical and subcortical areas. Although plasticity is a valuable trait that allows the human brain to rewire and recover from injury and sensory deprivation, it can lead to tinnitus as an unwanted side effect. In this large fMRI study, we provide evidence that tinnitus is related to a more conservative form of reorganization than in hearing loss without tinnitus. This result contrasts with the previous notion that tinnitus is related to excessive reorganization. As a consequence, treatments for tinnitus may need to enhance the cortical plasticity, rather than reverse it.

Received Aug. 26, 2019; revised Jan. 13, 2020; accepted Jan. 26, 2020.

Author contributions: E.A.K., C.P.L., and P.v.D. designed research; E.A.K. performed research; E.A.K. and R.J.R. analyzed data; R.J.R. contributed unpublished reagents/analytic tools; E.A.K. wrote the first draft of the paper; R.J.R., C.P.L., and P.v.D. edited the paper; E.A.K. wrote the paper.

This work was supported by Dorhout Mees Foundation, Netherlands Organisation for Scientific Research, American Tinnitus Association, William Demant Foundation, Heinsius-Houbolt Foundation, and Steunfonds Audiologie and Stichting Gehoorgestoorde Kind. We thank Dave Langers for sharing expertise.

The authors declare no competing financial interests.

C.P. Lanting's present address: Radboud University, Radboud University Medical Center, Department of Otorhinolaryngology, 6500 HB Nijmegen, The Netherlands.

Correspondence should be addressed to Elouise A. Koops at e.a.koops@umcg.nl.

https://doi.org/10.1523/JNEUROSCI.2083-19.2020

Copyright $\odot 2020$ the authors

\section{Introduction}

Peripheral damage causes plasticity to occur in the area of the CNS that corresponds to the loss of function. In the auditory domain, hearing loss instigates plasticity that results in changes in tonotopic maps, spontaneous activity, and neural synchronicity (Robertson and Irvine, 1989; Eggermont and Roberts, 2004). Tonotopic maps are a striking feature of the mammalian auditory cortex and underlie the representation of complex sounds, such as speech. This spatial separation of frequencies originates in the inner ear, where high frequencies are processed in the base of the cochlea and low frequencies in the apex. This separation is maintained from the cochlea to the auditory cortex (Brugge and Merzenich, 1973; Rauschecker et al., 1995). The tonotopic maps 
Table 1. Demographics and questionnaire scores of the three participants groups

\begin{tabular}{|c|c|c|c|}
\hline Groups & Controls & Hearing loss & Tinnitus + hearing loss \\
\hline Demographics & $N=38$ & $N=17$ & $N=35$ \\
\hline Sex & $20 \mathrm{M}, 18 \mathrm{~F}$ & $9 \mathrm{M}, 8 \mathrm{~F}$ & $29 \mathrm{M}, 6 \mathrm{~F}^{*},{ }^{* *}$ \\
\hline Mean age (years) & $46 \pm 13(22-67)$ & $61 \pm 10(33-75)^{*}$ & $59 \pm 10(26-73)^{*}$ \\
\hline HADS Anxiety & $4.5 \pm 3.4(0-11)$ & $3.1 \pm 3.7(0-11)$ & $5 \pm 3.5(0-12)$ \\
\hline HADS Depression & $3 \pm 3.1(0-8)$ & $2.7 \pm 2.9(0-10)$ & $4.5 \pm 3.9(0-16)$ \\
\hline HQ & $12.3 \pm 7.9(0-33)$ & $8.7 \pm 5.2(2-17)$ & $19.3 \pm 9.1(2-37)^{* * *}$ \\
\hline \multicolumn{4}{|l|}{ Tinnitus } \\
\hline Mean duration (years) & & & $12.3 \pm 8.6(1-33)$ \\
\hline \multirow{4}{*}{ Tinnitus pitch } & & & $2-4 \mathrm{kHz}(n=11)$ \\
\hline & & & $4-8 \mathrm{kHz}(n=3)$ \\
\hline & & & $\geq 8 \mathrm{kHz}(n=17)$ \\
\hline & & & $\bar{W}$ Wite noise $(n=4)$ \\
\hline
\end{tabular}

* The group differed significantly from the control group.

**The hearing loss groups differed significantly from one another at $p<0.001$.

can be disrupted by hearing loss, the most prevalent sensory deficit in the elderly population.

The presence of clinical hearing loss increases the chances of developing tinnitus, the perception of sound in the absence of an external source. To date, the specific pathophysiology involved in tinnitus remains elusive. However, the tinnitus pitch is often constrained to the frequency regions affected by hearing loss (Schecklmann et al., 2012; Shekhawat et al., 2014; Sereda et al., 2015; Keppler et al., 2017) or to the border of the intact hearing region (Moore and Vinay, 2010). These findings suggest that hearing loss and tinnitus are intricately related. Excessive or conservative tonotopic reorganization may differentiate between hearing loss with and without tinnitus.

Several papers have suggested a relation between hearing loss-induced tonotopic reorganization and tinnitus (Robertson and Irvine, 1989; Muhlnickel et al., 1998; Rauschecker, 1999; Eggermont and Roberts, 2004; Norena and Eggermont, 2005; Eggermont, 2006), but few have directly investigated this relation. In previous experimental work, it was observed that tonotopic map plasticity was linked to hearing loss but not to tinnitus (Weisz et al., 2005; Wienbruch et al., 2006; McMahon et al., 2016). In humans, tonotopic map reorganization was reported in one MEG study on tinnitus. A positive correlation was reported between the strength of the perceived tinnitus and the extent of cortical reorganization (Muhlnickel et al., 1998). In contrast, other studies reported no tonotopic plasticity related to tinnitus in humans (Langers et al., 2012) or animals (Kotak et al., 2005; Yang et al., 2011). Instead, these animal studies identified enhanced cortical excitation or reduced cortical inhibition in animals with binaural hearing loss and behavioral signs of tinnitus. The release from inhibition in the hearing loss affected area connects the tinnitus pitch with increased neuronal excitability (Yang et al., 2011). In general, it is not well established that tonotopic map plasticity is a cortical characteristic of tinnitus.

Animal models of cortical tonotopic reorganization indicate that receptive fields of neurons within the hearing loss-affected area shift toward the intact receptors (Rajan and Irvine, 1998; Eggermont and Komiya, 2000; Irvine et al., 2001; Muhlau et al., 2006). This reorganization causes a downward shift in the characteristic frequency of neurons, in both temporary and lasting hearing loss (Irvine et al., 2000; Norena and Eggermont, 2005, 2006), thus altering the tonotopic map. In contrast, not all animal studies on hearing loss found a downward shift in tonotopic maps, but instead reported increased excitability (Kotak et al., 2005) or decreased inhibition (Rajan, 1998) of the affected frequency regions. In humans, one MEG study reported a shift of the cortical responsive region toward the intact edge-frequency of the audiogram in hearing loss (Dietrich et al., 2001). In summary, different correlates of tonotopic plasticity have been reported in the literature on hearing loss and tinnitus, and the translation of animal models to human imaging is sparse, especially in tinnitus.

This large fMRI study examined the relation between hearing loss, tinnitus, and tonotopic reorganization with loudnessmatched sound stimuli in humans. Inclusion of participants with high-frequency hearing loss, both with and without tinnitus, allowed us to investigate to what extent reorganization is a consequence of hearing loss, and whether any reorganization is specifically related to tinnitus.

\section{Materials and Methods}

The study was approved, in accordance with the principles of the declaration of Helsinki (2013), by the medical ethical committee of the University Medical Center Groningen, The Netherlands. Written informed consent was obtained, and participants received reimbursement for their participation.

\section{Participants}

A total of 113 participants, both male and female, were included in a larger MRI study. In 90 participants, three complete functional runs were obtained. This resulted in 35 participants with hearing loss and tinnitus, 17 participants with hearing loss without tinnitus, and 38 healthy controls without hearing loss or tinnitus (Table 1). None of the participants was using hearing aids to compensate their hearing loss or ameliorate their tinnitus. Pure tone audiometry was performed in a sound attenuating booth to determine hearing thresholds for all participants at octave frequencies ranging from 0.125 to $8 \mathrm{kHz}$. Tinnitus pitch and loudness were estimated with a matching procedure. In addition, the participants completed the Tinnitus Handicap Inventory (McCombe et al., 2001), the Tinnitus Reactions Questionnaire (Wilson et al., 1991), the Hyperacusis Questionnaire (HQ) (Khalfa et al., 2002), and the Hospital Anxiety and Depression Scale (HADS) (Zigmond and Snaith, 1983).

Group differences were tested with a $\chi^{2}$ test of independence for the variable sex, and a three-group ANOVA followed-up by independent pairwise $t$ tests for the variable age. The questionnaire scores were assessed by means of a Kruskal-Wallis test and followed up by a pairwise Mann-Whitney test. 


\section{Data acquisition}

All MRI data were obtained with a $3.0 \mathrm{~T}$ Philips Intera MRI scanner (Best), at the Neuro Imaging Center Groningen. The scanner was equipped with a SENSE 32-channel head coil. Both structural and functional images were obtained for each participant. The structural image was a whole-brain T1-weighted image (voxel size $1 \mathrm{~mm} \times 1 \mathrm{~mm} \times 1$ $\mathrm{mm}$ ). The functional images were acquired in a sparse imaging sequence (Hall et al., 1999), as single-shot EPI: 47 slices; no gap; scan matrix $72 \times 67$; descending slice order; TR of $10 \mathrm{~s}$, TE $22 \mathrm{~ms}$, flip angle $90^{\circ}$. For each participant, a total of three runs, of each 65 EPI volumes, were acquisitioned.

\section{Sound stimuli}

During the fMRI experiments, loudness-matched auditory stimuli were presented. Before the MRI session, participants performed a binaural loudness matching task in which the stimulus tones at $0.25,0.5,2,4$, and $8 \mathrm{kHz}$ were all matched in perceived loudness to a $1 \mathrm{kHz}$ tone at $40 \mathrm{~dB}$ SPL. This compensates for loudness distortion present in sensorineural hearing loss (Moore and Glasberg, 2004). In addition, studies indicate that sound-evoked cortical activation correlates better with loudness rather than the level of sound stimuli (Hall et al., 2001; Langers et al., 2007). A two alternative-forced-choice, 1-up-1-down loudness matching procedure was used to approximate equal loudness sensation over all frequencies. An interleaved staircase method was applied, with a maximum of 15 trials per frequency, 7 reversals, and a step size of $[10,5,5,3,3,1] \mathrm{dB}$ SPL. This method yielded an equal loudness contour for each participant.

\section{Procedure MRI}

The individually loudness-matched auditory stimuli were presented during the relatively silent scanner intervals in the sparse sampling protocol. The auditory stimuli were $245 \mathrm{~ms}$ in length and were repeated at a $4 \mathrm{~Hz}$ repetition rate. Every volume acquisition consisted of $7.5 \mathrm{~s}$ of sound stimulation with one frequency, followed by $2 \mathrm{~s}$ of scanning. In addition to the sound stimuli, there was a silence condition. Stimulus conditions were presented binaurally in a quasi-random order via an MR Confon Sound System (Baumgart et al., 1998). Sound levels in the MRI were calibrated with a B\&K 4134 microphone, inserted in the ear of a KEMAR dummy.

To control for effects of attention, participants were instructed to perform a visual valence task similar to the task used by Langers and van Dijk (2012). Participants were instructed that the sound stimuli were irrelevant and asked to concentrate on the visual task.

\section{Statistical analysis}

Data preprocessing

The fMRI data analysis was performed in MATLAB (version 2018a), and with the aid of SPM12 (Statistical Parametric Mapping). Functional images were preprocessed, realigned, and coregistered to the anatomic image, then normalized to fit a standard brain (MNI), and resliced to a voxel width of $2 \mathrm{~mm}$. With the use of a Gaussian filter, the images were smoothed with a Gaussian kernel with FWHM of $5 \mathrm{~mm}$. During preprocessing, a logarithmic transformation was applied to the fMRI volumes, to convert output to units of percentage signal change (Langers and van Dijk, 2012).

A second-level analysis was performed to assess the response to sound, voxel by voxel, on group level, by means of an $F$ test on the 6 coefficients of the sound-frequency related regressors. A minimum cluster size of $k>1000$ was used to exclude smaller activation clusters of no interest to tonotopic mapping. The remaining activation clusters were used to construct an ROI for further analyses ( $n=5141$ voxels).

\section{Group comparisons}

Group differences in median activation levels and corresponding Bayes Factors were calculated for each frequency. Differences in activation patterns between the groups were obtained by calculating the Euclidean distance per frequency, based on the mean signal change in all voxels:
A

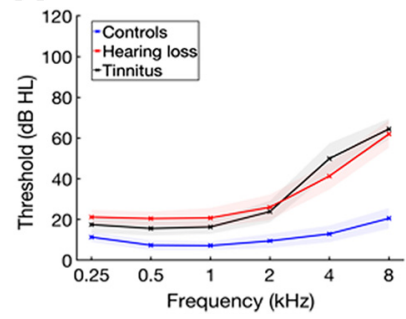

B

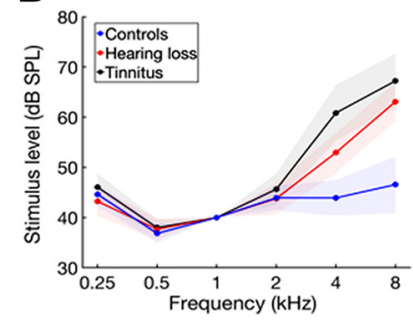

Figure 1. Hearing characteristics of participants. $A$, Audiometric thresholds used in the MRI scanning protocol are indicated here, with their corresponding SE. $\boldsymbol{B}$, During MRI scanning, stimuli were presented at loudness levels equal to the 40 phon loudness curve. All stimuli were thus matched in loudness to a $1 \mathrm{kHz}$ pure tone at $40 \mathrm{~dB}$ SPL. The average levels of the stimuli are depicted per group, for the six frequencies presented along with their corresponding SE.

$\mathrm{d}_{\mathrm{ab}}=$

$$
\sqrt{ }\left(\sum_{i}^{n}\left(x_{a i}-x_{b i}\right)^{2}\right)
$$

where $a$ and $b$ refer to the two groups being compared, and the sum is taken over all $n=5141$ voxels in the cortical ROIs. This distance was computed for each stimulus frequency. It is a measure of the difference in activation patterns between the groups $a$ and $b$. The voxels were assigned to the different frequencies according to their peak activation responsiveness. Permutation testing was performed to assess statistical significance of the group differences.

\section{Principal component analysis (PCA)}

In order to obtain a robust measure for tonotopic map changes, a PCA was performed by means of singular value decomposition, without centering (similar to Langers et al., 2012). The participant matrices $(5141 \times 6)$ were concatenated to form an aggregate matrix A of $462,690 \times 6$ (90 participants $\times 5141$ voxels $\times 6$ frequencies $)$. The principal components $\left(\mathrm{X}_{\mathrm{i}}\right)$ were extracted from this matrix A. Frequencywise analyses were performed on the aggregate matrix A, expressing percentage signal change instead of principal component loadings. The advantage of performing PCA on one concatenated matrix containing data of all participants is that all PCA-derived component maps are based on the same principal components and can therefore be compared across participants (Langers et al., 2014).

Assessment of the statistical significance of these principal component scores was done by calculating, for each pairwise group comparison, the Mahalanobis distance to quantify the magnitude of separation between the principal component clusters of the different groups. The method described here was coined by Goodpaster and Kennedy (2011). The Mahalanobis distance definition used was: $D_{M}(P C 1, P C 2)=$ $\sqrt{\mathrm{d}^{\prime} C_{W}^{-1} d}$, based on the median voxel response per participant. With $d$ expressed as the difference vector between the centroids of two groups according to $d=\left[C_{P C 12}-C_{P C 11}, C_{P C 22}-C_{P C 21}\right]$, and $C_{W}^{-1}$ as the pooled variance covariance matrix between two groups. To test whether the cluster separation was significant between groups, a Hotelling's $\mathrm{T}^{2}$ statistic was calculated, according to the following equation: $T^{2}=$ $\frac{n 1 n 2}{n 1+n 2} \mathrm{~d}^{\prime} C_{W}^{-1} \mathrm{~d}$. The $n$ values indicate the sample sizes of the two groups. $\mathrm{A}$ larger $\mathrm{T}^{2}$ statistic indicates a larger distance between the PCA score centroids of the two groups. Next, an $F$ test was performed and the $F$ value, the ratio of between versus within group variance, computed according to: $F(p, n 1+\mathrm{n} 2-p-1)=\frac{n 1+n 2-p-1}{p(n 1+n 2-2)} T^{2}$, with $p$ being the discriminator variables (the two PCs). The critical $F$ value was determined in a look-up table, based on the numerator and denominator degrees of freedom at $\alpha=0.05$. This critical $F$ value determines whether the variance between the centroids of two groups is significant. Finally, a $p$ value was calculated for each group comparison to determine if the probability of this finding is small enough to reject the null-hypothesis; that is, there are no differences in PC scores between the groups. 
A

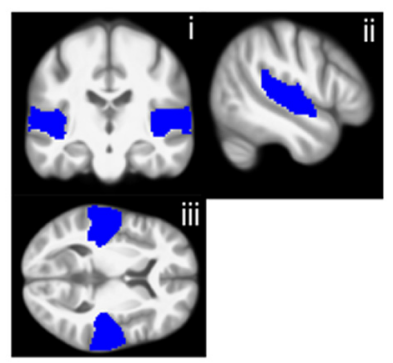

C

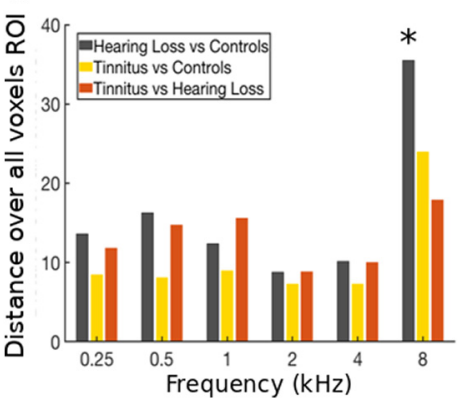

B

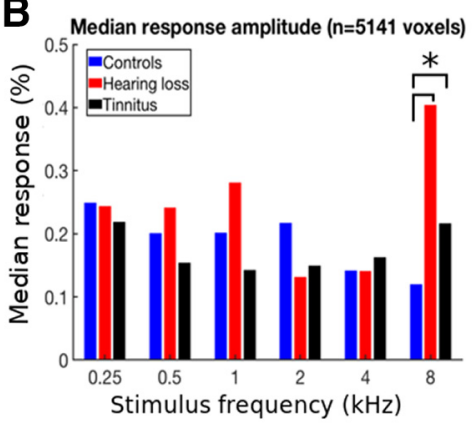

D

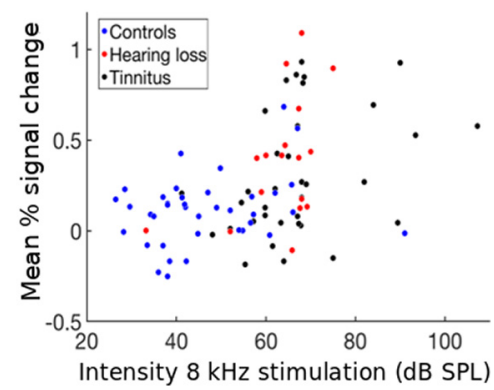

Figure 2. Sound-evoked activation levels. $\boldsymbol{A}$, ROls based on overall activated voxels $(n=5141)$ in response to sound, across all 90 participants. $\boldsymbol{B}$, Group-level responsiveness profile, based on percentage signal change in ROI voxels in response to the six presented frequencies. A significant difference $(p<0.05)$ in the responsiveness levels was observed for both hearing loss groups, with and without tinnitus, compared with the control group, in response to $8 \mathrm{kHz}$ stimulation $(p=0.02$ and $p=0.003$, respectively), indicated by the asterisk. However, significance remains only for the hearing loss group without tinnitus when corrected for multiple comparisons (Bonferroni-corrected $0.05 / 6=0.008$ ). C, Euclidian distance between response profiles of participant groups, per frequency. The distance was computed using the response amplitudes of all voxels as spatial response profile. A smaller distance indicates more similar voxel responses on that frequency. The statistical significance of the distances was determined by means of permutation testing $(n=50,000)$. The distance between hearing loss without tinnitus and controls is significant for $8 \mathrm{kHz}(p, 0.0028$, Bonferroni-corrected), indicated by the asterisk. $\boldsymbol{D}$, Mean percentage signal change per group during 8 $\mathrm{kHz}$ stimulation. Per participant, the level of stimulation (in $\mathrm{dB} \mathrm{SPL}$ ) at $8 \mathrm{kHz}$ is plotted against the mean percentage signal change over all voxels in the ROl. Although the absolute and mean highest percentage signal change occurred in the hearing loss group, the highest levels of stimulation were applied in the tinnitus group.

\section{Results}

To assess differences in cortical responsiveness to sounds, sparse-sampled sound-evoked cortical activation was obtained for 38 control participants, 17 participants with hearing loss but without tinnitus, and 35 participants with hearing loss and tinnitus (Table 1). The participant groups with hearing loss were well matched on hearing thresholds (Fig. $1 A$ ). There are no significant differences between the hearing loss groups at the included octave frequencies, except at $500 \mathrm{~Hz}$ (Mann-Whitney test, $p=0.05$ ). The control group differs significantly from both hearing loss groups on all frequencies $(p<0.05)$. Accordingly, the mean equal loudness contours of the stimuli indicate that both hearing loss groups needed higher sound intensities to perceive equal loudness at 4 and $8 \mathrm{kHz}$ compared with the control group (Fig. 1B).

The groups differ significantly in terms of sex distribution $(p=0.014)$, with a significantly larger proportion of men in the tinnitus group. A significant difference in age $\left(F_{(14,72)}, p<0.001\right)$ exists between the groups, which is due to the difference between the tinnitus and control group $(p<0.001)$ and the hearing loss and control group $(p<0.001)$. There is no significant difference in age $(p=0.529)$ between the groups with hearing loss, with or without tinnitus. HADS subscales did not show significant group differences. HQ score distributions differed significantly between the groups $(p=0.001)$. Post hoc testing showed that the hearing loss and control groups did not differ significantly $(p=0.133)$, in contrast to the tinnitus and hearing loss $(p<$ $0.001)$ and the tinnitus and control comparisons $(p=0.007)$. In the hearing loss group with tinnitus, 5 participants had HQ scores that could indicate a reduced tolerance to sound; the exclusion of these participants did not alter any of the measures displayed, and hence they were included in the analyses.

\section{Sound-evoked activation}

To determine the sound-evoked cortical activation, ROIs were constructed based on the overall significantly activated voxels in response to sound, across all 90 participants (familywise error $<0.05$, cluster size $k>1000$; Fig. $2 A)$. This was done by weighing all 6 sound-stimulus regressors equally in an omnibus $F$ test. All subsequent second-level analyses were performed on these 5141 voxels corresponding roughly to the bilateral auditory cortices. For each stimulus frequency, the average signal change was computed across all voxels in the ROI. The cortical response to $8 \mathrm{kHz}$ is significantly larger in the tinnitus (Mann-Whitney test, $p=0.025, Z=2.25$, Bayes Factors $\left.\left[\mathrm{BF}_{10}\right]=1.82\right)$ and the hearing loss $\left(p=0.003, Z=2.94, \mathrm{BF}_{10}=5.24\right)$ groups compared with the control group, and this response is large compared with voxels with different preferred frequencies (Fig. 2B). Nevertheless, the $\mathrm{BF}_{10}$ indicates that this effect is more robust for the hearing loss group without tinnitus. A one-way ANOVA indicated that the differences in percentage signal change between participants was not explained by age $\left(F_{(2,41)}=1.167, p=0.341\right)$ or sex differences $\left(F_{(2,1)}=0.287, p=0.599\right)$ but confirmed the significant differences for group $\left(F_{(2,2)}=4.17, p=0.026\right)$.

Similarity in cortical activation patterns was investigated by means of a Euclidean distance measure, calculated for all three group comparisons. A small Euclidean distance between two groups implies that their cortical activation patterns are similar. The cortical activation patterns of the group with tinnitus and the control group are most similar to each other, except at $8 \mathrm{kHz}$ (Fig. 2C). At $8 \mathrm{kHz}$, the activation pattern of the hearing loss group without tinnitus diverged strongly, and significantly $(p<0.0028)$, from the control group. In the group with tinnitus, a similar but nonsignificant shift was observed.

Additional analyses were performed to investigate whether the highest responsiveness levels at $8 \mathrm{kHz}$ could be explained by the highest levels of stimulation. Because of the presence of highfrequency hearing loss, both hearing loss groups with and without tinnitus were stimulated at higher intensities in the high frequencies than the control group. For each participant, the percentage signal change in response to $8 \mathrm{kHz}$ stimulation was plotted against the intensity of stimulation (Fig. 2D). The highest 
stimulation levels occurred in the tinnitus group, whereas the highest percentage signal change occurred in the hearing loss group. The overrepresentation of high frequencies persists when only moderate hearing losses ( $\leq 60 \mathrm{~dB}$ hearing loss at $8 \mathrm{kHz}$ ) or mild stimuli levels $(<1$ SD control mean) are considered. This suggests that the higher levels of activation are not the direct result from higher levels of stimulation.

\section{PCA}

To obtain robust tonotopic response maps, PCA was used. The first and second principal component's response profiles, over all voxels, were obtained by an analysis that included all three participant groups (Fig. 3A,B). We included the first two principal components, with the first principal component explaining $73 \%$ of the variance in the signal and the second component an additional $11 \%$. The first principal component reflects overall responsiveness to sound stimulation (Fig. $3 A$ ), as a direct comparison to the overall activation confirmed.

The tonotopic maps could be inferred from the cascaded response profile of the second principal component, which shows a stagewise increase from negative loadings on low frequencies to positive loadings on high frequencies (Fig. $3 B$ ). The aggregate responses were portioned into individual spatial response maps to compute the average group maps (Fig. $3 C$ ). This showed that the high frequencies are more dominant in the spatial frequency group maps of both hearing loss groups, compared with the controls. This high-frequency dominance is strongest for the hearing loss group without tinnitus (Fig. 3C).

Assessment of the differences in principal component scores of the first and the second principal component was done by calculating the Mahalanobis distance, Hotelling's $T^{2}, F$ statistics, and $p$ values (Table 2 ). These analyses showed that the principal component scores, both for the first and the second principal components, of the hearing loss group without tinnitus were significantly different from those of the control group, as indicated by the critical $F$ value and $p$ value $(p=0.012)$ at a level of $p$ for multiple comparisons $(p=0.0167)$. The difference between the principal component scores of the hearing loss group with tinnitus and the control group nearly reached significance $(p=0.0175)$, whereas the hearing loss groups, with and without tinnitus, were not significantly different from one another $(p=0.5864)$.

\section{Discussion}

Our findings show that functional reorganization of the auditory cortex is less pronounced in hearing loss with tinnitus than in hearing loss without tinnitus. Both the response amplitudes and the tonotopic map characteristics in participants with tinnitus were intermediate to those of normal hearing control participants and hearing loss participants without tinnitus. Thus, the
Table 2. Summary of pairwise cluster separations of the first and second component given by Mahalanobis distances, Hotelling's $T^{2}$ statistics, $F$ statistics, and $p$ values

\begin{tabular}{|c|c|c|c|c|}
\hline & $\begin{array}{l}\text { Mahalanobis } \\
\text { distance }\end{array}$ & $\begin{array}{l}\text { Hotelling's } \\
\mathrm{T}^{2} \text { statistic }\end{array}$ & $F$ & $p$ \\
\hline Hearing loss versus controls & 0.9145 & 9.82 & 4.82 & $0.0120^{*}$ \\
\hline $\begin{array}{l}\text { Tinnitus }+ \text { hearing loss } \\
\text { versus controls }\end{array}$ & 0.6912 & 8.70 & 4.29 & 0.0175 \\
\hline $\begin{array}{c}\text { Tinnitus }+ \text { hearing loss } \\
\text { versus hearing loss }\end{array}$ & 0.3102 & 1.10 & 0.54 & 0.5864 \\
\hline
\end{tabular}

* The groups differed significantly from one another on a $p$ level corrected for multiple comparisons.

reorganization is a consequence of hearing loss and is more conservative in hearing loss with tinnitus. In other words, the presence of tinnitus in hearing loss appears not to relate to excessive cortical plasticity but rather to more diminished adaptation than in hearing loss alone.

The increased response amplitudes in both hearing loss groups were present only at $8 \mathrm{kHz}$. At this frequency, the hearing loss was largest, of the frequencies tested, for the majority of our hearing loss participants (75\%). This is typical for (age-related) high-frequency sensorineural hearing loss (Gates and Mills, 2005). It is worth noting that the stimuli in our experiments were loudness-matched across frequency for each participant individually. This loudness matching ensured that all stimuli were audible and perceived as equally loud, regardless of raised hearing thresholds. Consequently, the stimulus intensity levels at higher sound frequencies were increased in the hearing loss groups, with and without tinnitus, compared with the normal hearing participants (Fig. 1). In the tinnitus group, this effect was not related to the tinnitus frequency. Although most tinnitus participants had high-frequency tinnitus (Table 1), the tinnitus pitch was not significantly correlated with the frequency eliciting the highest percentage signal change $(R=-0.217, p=0.276)$. The 
lack of significant correlation suggests that the increased responsiveness at $8 \mathrm{kHz}$ is not related to the tinnitus itself but rather to the accompanying hearing loss. This is in line with the finding that this increase in responsiveness is present in both the hearing loss group with and without tinnitus.

Generally, the stimulus levels were similar in the two hearing loss groups, although in some instances the intensities were larger in the hearing loss group with tinnitus (Fig. 2C; data points at $80-110 \mathrm{~dB}$ SPL). Hence, it is quite remarkable that the cortical responses were largest in the hearing loss group without tinnitus, despite that the stimulus intensities did not surpass those of the hearing loss group with tinnitus. Similarly, the largest differences in the tonotopic map were found when contrasting the hearing loss group without tinnitus to the normal hearing participants. Conversely, the tonotopic map of the hearing loss participants with tinnitus was more similar to those of normal hearing participants (Figs. 2, 3). Since these differences cannot simply be accounted for by the differences in stimulus intensities, it may reflect different degrees of (re)organization of the auditory system for participants with hearing loss and tinnitus compared with those without tinnitus.

The majority of tinnitus-related fMRI studies included participants with normal hearing thresholds or mild hearing losses. The results across these studies are variable. Gu et al. (2010) reported elevated auditory cortex activation in tinnitus participants with normal hearing. Unfortunately, their hyperacusis controlled design resulted in rather small participant groups $(n=7$ with tinnitus, $n=5$ without tinnitus). In a similar fMRI study by Langers et al. (2012), cortical response amplitudes were similar between normal hearing participants with and without tinnitus, expect for a small region in the lateral portion of left Heschl's gyrus. Similarly, Lanting et al. (2008) reported no differences in cortical response amplitudes in relation to unilateral tinnitus and mild to moderate hearing loss. In contrast, Hofmeier et al. (2018) showed a pronounced reduction of the cortical responses in tinnitus participants with mild hearing loss in a study that excluded hyperacusis.

The present study included participants with moderate to profound high-frequency hearing loss. In both hearing loss groups, with and without tinnitus, an increased responsiveness to $8 \mathrm{kHz}$ stimulation was observed compared with the normal hearing control group. These findings are in line with Ghazaleh et al. (2017), who reported no tinnitus-related differences in tonotopic map characteristics in participants with unilateral hearing loss and tinnitus. Boyen et al. (2014) also found no differences in cortical responses between hearing loss with and without tinnitus. Although the hearing loss in the Hofmeier et al. (2018) study was very mild (up to $40 \mathrm{~dB}$ per frequency), the results are very similar to that of the current study. There is no obvious explanation for the variability across these studies; however, the studies with larger participant groups (Lanting et al., 2008; Langers et al., 2012; Hofmeier et al., 2018) suggest that response amplitudes are either similar of reduced in tinnitus.

The reduced sound-evoked cortical amplitudes in hearing loss with tinnitus (Fig. 2B) (Hofmeier et al., 2018), in comparison with hearing loss without tinnitus, have been interpreted as a failure to increase response gain (Knipper et al., 2013; Hofmeier et al., 2018). This failure to increase response gain in the presence of heightened spontaneous activity presumably results in tinnitus. The cortical inability in tinnitus to adapt sufficiently to hearing loss finds a rationale in reduced levels of Arc, a cytoskeletal protein involved in long-term synaptic plasticity (Nikolaienko et al., 2018), as reported in the auditory cortex of tinnitus animals (Tan et al., 2007; Rüttiger et al., 2013). Whereas, generally, Arc is mobilized after inducing hearing loss (Kapolowicz and Thompson, 2016), the expression of Arc is significantly reduced in animals that develop tinnitus (Rüttiger et al., 2013). These findings support the notion that, at a cortical level, tinnitus, in the presence of hearing loss, is associated with insufficient adaptation to hearing loss.

The enhanced representation of high frequencies in hearing loss appears to contrast with some animal models of tonotopic reorganization. Several animal studies reported the absence of high-frequency responsiveness in the auditory cortex and overrepresentation of low frequencies in animals with induced highfrequency hearing loss (Rajan and Irvine, 1998; Irvine et al., 2000; Norena and Eggermont, 2005). The differences between these animal studies and our human data presumably relate to differences in techniques used to assess cortical neural activity. The animal models were based on best or characteristic frequencies of cortical neurons, which are measured with nearthreshold stimuli. This method is especially informative of the spatial localization and extent of the neuronal population that preferentially respond to a certain frequency. In our study, we measured BOLD responses at suprathreshold levels; the BOLD response is informative of the cortical area that responds to sound stimulation as well as the intensity or amplitude of this response. Therefore, these findings may not contrast each other but instead investigate a different aspect of the cortical responses to sound.

Finally, although our results show group differences in the auditory cortex, it is not clear whether these differences arise due to changes in the function of the cochlea or the brain. Naturally, sensorineural hearing loss involves cochlear pathology. However, the differences observed between the hearingimpaired participants with tinnitus and those without tinnitus may be due to both cochlear and central differences. Recent evidence suggests that tinnitus is associated with both reduced ribbon synapse density in the cochlea (Rüttiger et al., 2013; Zhang et al., 2014) and reduced ARC expression in the cortex (Rüttiger et al., 2013; Singer et al., 2013). With the measures of the present study (i.e., pure tone audiometry and MRI), it is not possible to identify differences in cochlear pathology between the hearing loss groups.

\section{Limitations}

In earlier studies, it was described that, with increasing age, stronger sound-evoked responses were observed in the auditory cortex (Profant et al., 2015; Recanzone, 2018). To investigate whether the observed group differences in the present study were not caused by age differences, we plotted per group the age of participants against their high-frequency evoked cortical activation to observe any correlation. This demonstrated that none of the groups showed any significant or near-significant correlation between age and high-frequency evoked cortical activation levels (tinnitus + hearing loss $R=-0.105, p=0.547$; hearing loss $R=0.119, p=0.650$; controls $=0.246, p=0.137$ ). However, it must be noted that our hearing loss group without tinnitus has fewer younger people compared with the hearing loss group with tinnitus.

In conclusion, hearing loss was associated with higher levels of sound-evoked cortical responsiveness, and this increase was most pronounced in the group with hearing loss but without tinnitus. Both in terms of response amplitudes and tonotopic map characteristics, the participants with hearing loss and tinnitus 
appear intermediate to the controls and the hearing loss participants without tinnitus. This suggests that tinnitus is related to an incomplete form of central compensation to hearing loss, rather than excessive adaptation. As a consequence, treatments for tinnitus may need to enhance the cortical plasticity, rather than reversing it.

\section{References}

Baumgart F, Kaulisch T, Tempelmann C, Gaschler-Markefski B, Tegeler C, Schindler F, Stiller D, Scheich H (1998) Electrodynamic headphones and woofers for application in magnetic resonance imaging scanners. Med Phys 25:2068-2070.

Boyen K, de Kleine E, van Dijk P, Langers DR (2014) Tinnitus-related dissociation between cortical and subcortical neural activity in humans with mild to moderate sensorineural hearing loss. Hear Res 312:48-59.

Brugge JF, Merzenich MM (1973) Responses of neurons in auditory cortex of the macaque monkey to monaural and binaural stimulation. J Neurophysiol 36:1138-1158.

Dietrich V, Nieschalk M, Stoll W, Rajan R, Pantev C (2001) Cortical reorganization in patients with high frequency cochlear hearing loss. Hear Res 158:95-101.

Eggermont JJ (2006) Cortical tonotopic map reorganization and its implications for treatment of tinnitus. Acta Otolaryngol Suppl 126:9-12.

Eggermont JJ, Komiya H (2000) Moderate noise trauma in juvenile cats results in profound cortical topographic map changes in adulthood. Hear Res 142:89-101.

Eggermont JJ, Roberts LE (2004) The neuroscience of tinnitus. Trends Neurosci 27:676-682.

Gates GA, Mills JH (2005) Presbycusis. Lancet 366:1111-1120.

Ghazaleh N, Zwaag WV, Clarke S, Ville DV, Maire R, Saenz M (2017) Highresolution fMRI of auditory cortical map changes in unilateral hearing loss and tinnitus. 30:685-697.

Goodpaster AM, Kennedy MA (2011) Quantification and statistical significance analysis of group separation in NMR-based metabonomics studies. Chemometr Intell Lab Syst 109:162-170.

Gu JW, Halpin CF, Nam EC, Levine RA, Melcher JR (2010) Tinnitus, diminished sound-level tolerance, and elevated auditory activity in humans with clinically normal hearing sensitivity. J Neurophysiol 104:3361-3370.

Hall DA, Haggard MP, Akeroyd MA, Palmer AR, Summerfield AQ, Elliott MR, Gurney EM, Bowtell RW (1999) 'Sparse' temporal sampling in auditory fMRI. Hum Brain Mapp 7:213-223.

Hall DA, Haggard MP, Summerfield AQ, Akeroyd MA, Palmer AR, Bowtell RW (2001) Functional magnetic resonance imaging measurements of sound-level encoding in the absence of background scanner noise. J Acoust Soc Am 109:1559-1570.

Hofmeier B, Wolpert S, Aldamer ES, Walter M, Thiericke J, Braun C, Zelle D, Rüttiger L, Klose U, Knipper M (2018) Reduced sound-evoked and resting-state BOLD fMRI connectivity in tinnitus. Neuroimage Clin 20:637-649.

Irvine DR, Rajan R, Brown M (2001) Injury- and use-related plasticity in adult auditory cortex. Audiol Neurootol 6:192-195.

Irvine DR, Rajan R, McDermott HJ (2000) Injury-induced reorganization in adult auditory cortex and its perceptual consequences. Hear Res 147:188199.

Kapolowicz MR, Thompson LT (2016) Acute high-intensity noise induces rapid Arc protein expression but fails to rapidly change GAD expression in amygdala and hippocampus of rats: effects of treatment with D-cycloserine. Hear Res 342:69-79.

Keppler H, Degeest S, Dhooge I (2017) The relationship between tinnitus pitch and parameters of audiometry and distortion product otoacoustic emissions. J Laryngol Otol 131:1017-1025.

Khalfa S, Dubal S, Veuillet E, Perez-Diaz F, Jouvent R, Collet L (2002) Psychometric normalization of a hyperacusis questionnaire. ORL J Otorhinolaryngol Relat Spec 64:436-442.

Knipper M, Van Dijk P, Nunes I, Rüttiger L, Zimmermann U (2013) Advances in the neurobiology of hearing disorders: recent developments regarding the basis of tinnitus and hyperacusis. Prog Neurobiol 111: $17-33$.
Kotak VC, Fujisawa S, Lee FA, Karthikeyan O, Aoki C, Sanes DH (2005) Hearing loss raises excitability in the auditory cortex. J Neurosci 25:3908-3918.

Langers DR, van Dijk P (2012) Mapping the tonotopic organization in human auditory cortex with minimally salient acoustic stimulation. Cereb Cortex 22:2024-2038.

Langers DR, van Dijk P, Schoenmaker ES, Backes WH (2007) fMRI activation in relation to sound intensity and loudness. Neuroimage 35:709718.

Langers DR, de Kleine E, van Dijk P (2012) Tinnitus does not require macroscopic tonotopic map reorganization. Front Syst Neurosci 6:2.

Langers DR, Krumbholz K, Bowtell RW, Hall DA (2014) Neuroimaging paradigms for tonotopic mapping: I. The influence of sound stimulus type. Neuroimage 100:650-662.

Lanting CP, De Kleine E, Bartels H, Van Dijk P (2008) Functional imaging of unilateral tinnitus using fMRI. Acta Otolaryngol 128:415-421.

McCombe A, Baguley D, Coles R, McKenna L, McKinney C, Windle-Taylor P, British Association of Otolaryngologists, Head and Neck Surgeons (2001) Guidelines for the grading of tinnitus severity: the results of a working group commissioned by the British Association of Otolaryngologists, Head and Neck Surgeons, 1999. Clin Otolaryngol Allied Sci 26:388-393.

McMahon CM, Ibrahim RK, Mathur A (2016) Cortical reorganisation during a 30-week tinnitus treatment program. PLoS One 11:e0148828.

Moore BC, Glasberg BR (2004) A revised model of loudness perception applied to cochlear hearing loss. Hear Res 188:70-88.

Moore BC, Vinay S (2010) The relationship between tinnitus pitch and the edge frequency of the audiogram in individuals with hearing impairment and tonal tinnitus. Hear Res 261:51-56.

Muhlau M, Rauschecker JP, Oestreicher E, Gaser C, Rottinger M, Wohlschlager AM, Simon F, Etgen T, Conrad B, Sander D (2006) Structural brain changes in tinnitus. Cereb Cortex 16:1283-1288.

Muhlnickel W, Elbert T, Taub E, Flor H (1998) Reorganization of auditory cortex in tinnitus. Proc Natl Acad Sci USA 95:10340-10343.

Nikolaienko O, Patil S, Eriksen MS, Bramham CR (2018) Arc protein: a flexible hub for synaptic plasticity and cognition. Semin Cell Dev Biol 77:33-42.

Norena AJ, Eggermont JJ (2005) Enriched acoustic environment after noise trauma reduces hearing loss and prevents cortical map reorganization. J Neurosci 25:699-705.

Norena AJ, Eggermont JJ (2006) Enriched acoustic environment after noise trauma abolishes neural signs of tinnitus. Neuroreport 17:559563.

Profant O, Tintěra J, Balogová Z, Ibrahim I, Jilek M, Syka J (2015) Functional changes in the human auditory cortex in ageing. PLoS One 10: $\mathrm{e} 0116692$.

Rajan R (1998) Receptor organ damage causes loss of cortical surround inhibition without topographic map plasticity. Nat Neurosci 1:138-143.

Rajan R, Irvine DR (1998) Neuronal responses across cortical field A1 in plasticity induced by peripheral auditory organ damage. Audiol Neurootol 3:123-144.

Rauschecker JP (1999) Auditory cortical plasticity: a comparison with other sensory systems. Trends Neurosci 22:74-80.

Rauschecker JP, Tian B, Hauser M (1995) Processing of complex sounds in the macaque nonprimary auditory cortex. Science 268:111-114.

Recanzone G (2018) The effect of ageing on auditory cortical function. Hear Res 366:99-105.

Robertson D, Irvine DR (1989) Plasticity of frequency organization in auditory cortex of guinea pigs with partial unilateral deafness. J Comp Neurol 282:456-471.

Rüttiger L, Singer W, Panford-Walsh R, Matsumoto M, Lee SC, Zuccotti A, Zimmermann U, Jaumann M, Rohbock K, Xiong H, Knipper M (2013) The reduced cochlear output and the failure to adapt the central auditory response causes tinnitus in noise exposed rats. PLoS One 8:e57247.

Schecklmann M, Vielsmeier V, Steffens T, Landgrebe M, Langguth B, Kleinjung T (2012) Relationship between audiometric slope and tinnitus pitch in tinnitus patients: insights into the mechanisms of tinnitus generation. PLoS One 7:e34878.

Sereda M, Edmondson-Jones M, Hall DA (2015) Relationship between tinnitus pitch and edge of hearing loss in individuals with a narrow tinnitus bandwidth. Int J Audiol 54:249-256. 
Shekhawat GS, Searchfield GD, Stinear CM (2014) The relationship between tinnitus pitch and hearing sensitivity. Eur Arch Otorhinolaryngol 271:41-48

Singer W, Zuccotti A, Jaumann M, Lee SC, Panford-Walsh R, Xiong H, Zimmermann U, Franz C, Geisler HS, Köpschall I, Rohbock K, Varakina K, Verpoorten S, Reinbothe T, Schimmang T, Rüttiger L, Knipper M (2013) Noise-induced inner hair cell ribbon loss disturbs central ARC mobilization: a novel molecular paradigm for understanding tinnitus. Mol Neurobiol 47:261-279.

Tan J, Rüttiger L, Panford-Walsh R, Singer W, Schulze H, Kilian SB, Hadjab S, Zimmermann U, Köpschall I, Rohbock K, Knipper M (2007) Tinnitus behavior and hearing function correlate with the reciprocal expression patterns of BDNF and Arg3.1/arc in auditory neurons following acoustic trauma. Neuroscience 145:715-726.
Weisz N, Wienbruch C, Dohrmann K, Elbert T (2005) Neuromagnetic indicators of auditory cortical reorganization of tinnitus. Brain 128:2722-2731.

Wienbruch C, Paul I, Weisz N, Elbert T, Roberts LE (2006) Frequency organization of the $40 \mathrm{~Hz}$ auditory steady-state response in normal hearing and in tinnitus. Neuroimage 33:180-194.

Wilson PH, Henry J, Bowen M, Haralambous G (1991) Tinnitus Reaction Questionnaire. J Speech Lang Hear Res 34:197- 201.

Yang S, Weiner BD, Zhang LS, Cho SJ, Bao S (2011) Homeostatic plasticity drives tinnitus perception in an animal model. Proc Natl Acad Sci USA 108:14974-14979.

Zhang FY, Xue YX, Liu WJ, Yao YL, Ma J, Chen L, Shang XL (2014) Changes in the numbers of ribbon synapses and expression of RIBEYE in salicylate-induced tinnitus. Cell Physiol Biochem 34:753-767.

Zigmond AS, Snaith RP (1983) The hospital anxiety and depression scale. Acta Psychiatr Scand 67:361-370. 